\title{
CONNECTION MATRIX PAIRS
}

\author{
DAVID RICHESON \\ Department of Mathematics, Northwestern University \\ 2033 Sheridan Rd., Evanston, Illinois 60208-2730, U.S.A. \\ E-mail: richeson@math.nwu.edu
}

\begin{abstract}
We discuss the ideas of Morse decompositions and index filtrations for isolated invariant sets for both single-valued and multi-valued maps. We introduce the definition of connection matrix pairs and present the theorem of their existence. Connection matrix pair theory for multi-valued maps is used to show that connection matrix pairs obey the continuation property. We conclude by addressing applications to numerical analysis. This paper is primarily an overview of the papers [R1] and [R2].
\end{abstract}

1. Introduction. In [F1] Franzosa proves the existence of connection matrices for Morse decompositions of isolated invariant sets for flows. This tool has proved very useful for detecting connections between Morse sets. Moreover, with the development of transition matrices, the connection matrix has proved useful for detecting heteroclinic bifurcations between Morse sets. The power of the connection matrix lies in its computability; like the Conley index, the connection matrix has the continuation property [F2].

Much of the recent research is directed towards the discrete Conley index. In this article we survey results generalizing the connection matrix to the discrete case. In [R1] the author proves the existence of a pair of matrices, the connection matrix pair, which generalizes the single connection matrix for flows to the case of a single-valued continuous map. Then, in [R2] connection matrix pairs are generalized to the case of multi-valued maps. Among other applications, this result proves that connection matrix pairs possess the continuation property.

Below we summarize the connection matrix pair results for multi-valued maps. Because most applications will be single-valued maps this generalization may seem to be an unnecessary and indulgent. Indeed, one may question why we develop the theory for multi-valued maps at all. It is not purely an academic generalization. First, with the

1991 Mathematics Subject Classification: Primary 58F12; Secondary 58F30, 58F14.

Key words and phrases: connection matrix pairs, Conley index, Morse decomposition, index filtrations, multi-valued maps.

The paper is in final form and no version of it will be published elsewhere. 
multi-valued results in place one finds that the continuation property of the Conley index is transparent. In the same vein we show that there exist stable index filtrations filtrations which are robust under small perturbations of the map. Lastly, because of the rise of computer assisted proofs, there has arisen a need for results about multi-valued maps. Below we indicate how Morse decompositions and connection matrix pairs can be used in numerical analysis. Moreover, all definitions and theorems below are still valid for single-valued maps. If definitions can be significantly simplified when one considers single-valued maps the author will make note of this.

2. Discrete multi-valued dynamical systems. Consider locally compact metric spaces $X$ and $Y$. We denote the power sets of $X$ and $Y$ by $\mathscr{P}(X)$ and $\mathscr{P}(Y)$. We say that $F: \mathscr{P}(X) \rightarrow \mathscr{P}(Y)$ is a multi-valued map provided for all $A, B \in \mathscr{P}(X)$,

$$
F(A \cup B)=F(A) \cup F(B)
$$

and

$$
F(\emptyset)=\emptyset \text {. }
$$

Notice that we do allow the possibility $F(A)=\emptyset$ for $A \neq \emptyset$. If $F: \mathscr{P}(X) \rightarrow \mathscr{P}(Y)$ is a multi-valued map then we may define another multi-valued map $F^{-1}: \mathscr{P}(Y) \rightarrow \mathscr{P}(X)$ by

$$
F^{-1}(A):=\{x \in X: F(x) \cap A \neq \emptyset\} .
$$

It is important to note that, despite the notation, $F^{-1}$ is not the inverse map to $F$. It is the case that $F\left(F^{-1}(A)\right) \subset A$ and that $\left(F^{-1}\right)^{-1}=F$.

If $F: \mathscr{P}(X) \rightarrow \mathscr{P}(X)$ is a self-map then we may define $F^{n}: \mathscr{P}(X) \rightarrow \mathscr{P}(X)$ for $n \in \mathbf{Z}$. We define $F^{0}=i d$ and $F^{-1}$ as above then require $F^{n+m}(A)=F^{n}\left(F^{m}(A)\right)$ where $n m \geq 0$.

Clearly, every multi-valued map can be completely described by its action on single points or, more precisely, sets consisting of a single point

$$
F(A)=\bigcup_{x \in A} F(x)
$$

Moreover, $F$ is often defined in this way. Thus, in the literature one often finds multivalued maps defined as maps from a space to the power set of another space $X \rightarrow \mathscr{P}(Y)$. This notation should cause no confusion.

A multi-valued map $F: \mathscr{P}(X) \rightarrow \mathscr{P}(Y)$ is said to be upper semicontinuous if $F^{-1}(A)$ is closed whenever $A$ is closed, or equivalently, $\{x \in X: F(x) \subset U\}$ is open whenever $U$ is open. The multi-valued map $F$ is continuous if $F^{-1}(U)$ is open whenever $U$ is open. If $F: \mathscr{P}(X) \rightarrow \mathscr{P}(X)$ is an upper semicontinuous map with the property that $F(x)$ is compact for all $x \in X$ then $F$ is called a discrete multi-valued semidynamical system (dmss). If, in addition, $F^{-1}$ is upper semicontinuous and takes compact values then $F$ is called a discrete multi-valued dynamical system (dmds). We have the following classes of maps.

$$
\begin{gathered}
\mathcal{F}(X):=\{F: \mathscr{P}(X) \rightarrow \mathscr{P}(X): F \text { is a dmss }\} \\
\mathcal{F}_{0}(X):=\{F \in \mathcal{F}(X): F \text { is continuous }\} .
\end{gathered}
$$


Clearly, not every $F \in \mathcal{F}(X)$ induces a map on cohomology. Thus, we must restrict our attention to a subclass of these maps. In [G1] and [G2] Górniewicz describes a class of functions called admissible maps or maps determined by a given morphism which may be used for computing Conley indices. We refer the reader to those references for the definitions. Examples of admissible maps are continuous single-valued maps and multivalued maps with compact acyclic values $\left(\tilde{H}_{*}(F(x))=0\right.$ for all $\left.x \in X\right)$. Also compositions of such maps are admissible. Let

$$
\begin{aligned}
\mathcal{G}(X) & :=\{F \in \mathcal{F}(X): F \text { is admissible }\} \\
\mathcal{G}_{0}(X) & :=\{F \in \mathcal{G}(X): F \text { is continuous }\}
\end{aligned}
$$

As was alluded to above, each continuous map $f: X \rightarrow X$ determines a dmss $F(x)=$ $\{f(x)\}$. If $f$ is proper, that is, $F^{-1}(K)$ is compact whenever $K$ is compact, then $F$ is a dmds. In particular, $F^{-1}(x)=f^{-1}(x)$.

We now present another multi-valued map. Suppose $G_{\lambda}$ is the fattening homotopy of $F$; that is, $G_{\lambda}:[0,1] \times \mathscr{P}(X) \rightarrow \mathscr{P}(X)$ is given by $G_{\lambda}(x)=\operatorname{cl}\left(B_{\lambda}(F(x))\right)$ where $B_{\lambda}(A)=\{x \in X: \operatorname{dist}(x, A)<\lambda\}$. We will call the multi-valued map $G_{\lambda}$ the $\lambda$-fattening map for $F$.

We call a subset $I \subset \mathbf{Z}$ an interval provided it is the intersection of a real interval with $\mathbf{Z}$. Suppose $I$ is an interval containing 0 . A single valued map $\sigma: I \rightarrow X$ is called a solution for $x \in X$ provided $\sigma(0)=x$ and $\sigma(n+1) \in F(\sigma(n))$ whenever $n, n+1 \in I$. If $I=\mathbf{Z}$ then $\sigma$ is called a full solution for $x$. Similarly, if $\mathbf{Z}^{+(-)} \subset I$ then $\sigma$ is called a full forward (backward) solution for $x$. A set $S$ is called invariant if there exists a full solution $\sigma_{x}: \mathbf{Z} \rightarrow S$ for each $x \in S$. For a set $N \subset X$ we $\operatorname{define~} \operatorname{Inv} N$ to be the maximal invariant subset of $N$. We impart a strong word of caution here; the term invariant may be slightly misleading, as an invariant set $S$ does not necessarily have the property that $F(S) \subset S$.

Definition 1. A compact subset $N \subset X$ is called an isolating neighborhood if $F(\operatorname{Inv} N) \subset \operatorname{Int} N$. If $N$ is an isolating neighborhood then $\operatorname{Inv} N$ is called an isolated invariant set.

REMARK 2. This definition is weaker than the one given by Kaczynski and Mrozek [KM1], but one can see that the crucial property of $N$ is that $x \in N$ cannot escape from $N$ under one iterate of $F$, thus all proofs in [KM1] still hold. This definition benefits from the absence of any mention of the metric.

REMARK 3. In some situations we may want this definition to be stronger. In particular, if $F$ is a dmds then this definition allows the possibility that an isolating neighborhood $N$ for $F$ not be an isolating neighborhood for $F^{-1}$. Thus, one may wish to require $F(\operatorname{Inv} N) \cup F^{-1}(\operatorname{Inv} N) \subset \operatorname{Int} N$. Since we are focusing on dmss we shall not make this assumption.

Multi-valued maps have properties which may be undesirable, or are at least unexpected, to those used to single-valued maps. For instance, there may exist a set $N$ with the property that $F^{i}(x) \cap N \neq \emptyset$ for $i=1, \ldots, n$ but there does not exist a solution $\sigma:\{0, \ldots, n\} \rightarrow N$ for $x$. This situation may be undesirable if $N$ is an isolating neigh- 
borhood or an invariant set. In fact, the map that we would like is $x \mapsto F(x) \cap N$. We denote this map by $F_{N}: \mathscr{P}(N) \rightarrow \mathscr{P}(N)$ and call it the restriction of $F$ to $N$. If $F$ is upper semicontinuous and $N$ is compact then $F_{N}$ is upper semicontinuous [KM1]. Using this definition we see that a set $S$ is invariant if and only if for all $x \in S, F_{S}(x) \neq \emptyset$ and $F_{S}^{-1}(x) \neq \emptyset$.

Definition 4. Let $S$ be an isolated invariant set for $F \in \mathcal{F}(X)$. An index pair for $S$ is a compact pair $(N, L)$ satisfying the following conditions:

1. $S=\operatorname{Inv}(\operatorname{cl}(N \backslash L)) \subset \operatorname{Int}(N \backslash L)$,

2. $F(L) \cap N \subset L$,

3. $F(N \backslash L) \subset N$,

The following result was proved by Kaczynski and Mrozek [KM1].

TheOREM 5. Let $F \in \mathcal{F}(X)$ and $W$ be a neighborhood of an isolated invariant set $S$. Then there exists an index pair $(N, L)$ for $S$ with $N \backslash L \subset W$.

We refer the reader to [KM1] for the details of defining the Conley index for discrete multi-valued dynamical systems. We now give a brief overview. Suppose $F \in \mathcal{G}(X)$ and $P=(N, L)$ is an index pair for an isolated invariant set $S$. The inclusion map

$$
i:(N, L) \rightarrow(N \cup F(L), L \cup F(L))
$$

induces an isomorphism in cohomology. Furthermore, since $F$ is admissible, the map

$$
F:(N, L) \rightarrow(N \cup F(L), L \cup F(L))
$$

induces a map on cohomology. Let $F_{P}: H^{*}(N, L) \rightarrow H^{*}(N, L)$ be the composition

$$
F_{P}:=F^{*} \circ\left(i^{*}\right)^{-1} \text {. }
$$

If we denote the Leray functor by $L$ then we define the (cohomological) Conley index of $S$ to be

$$
\mathrm{Con}^{*}(S)=\left(C H^{*}(S), \chi^{*}(S)\right):=L\left(H^{*}(N, L), F_{P}^{*}\right)
$$

As with the discrete single-valued map case, the Conley index consists of a graded abelian group and a distinguished automorphism. For our purposes we will consider the coefficients of all cohomology to lie in some field, thus $\operatorname{Con}^{*}(S)$ will actually consist of a graded vector space $C H^{*}(S)$ and a linear map, $\chi^{*}(S): C H^{*}(S) \rightarrow C H^{*}(S)$. That $\operatorname{Con}^{*}(S)$ is independent of index pair is proved by Kaczynski and Mrozek [KM1]. Furthermore, they show that the Conley index satisfies the additive and continuation properties.

We say that $G \in \mathcal{F}(X)$ is a selector for $F \in \mathcal{F}(X)$ if $G(x) \subset F(x)$ for all $x \in X$. We have the following useful result.

Proposition 6 [KM2]. Let $F \in \mathcal{F}(X), N$ be an isolating neighborhood and $P$ be an index pair for $\operatorname{Inv} N$. If $G \in \mathcal{F}(X)$ is a selector for $F$ then $N$ is an isolating neighborhood for $G, \operatorname{Inv}(N, G) \subset \operatorname{Inv}(N, F)$ and $P$ is an index pair for $G$. If $F, G \in \mathcal{G}(X)$ then $\operatorname{Con}^{*}(N, F) \cong \operatorname{Con}^{*}(N, G)$. 
3. Attractor-repeller pairs. Let $F \in \mathcal{F}(X)$ and $B \subset X$. We define the $\omega$-limit set of $B$ to be

and the $\alpha$-limit set of $B$ to be

$$
\omega(B)=\omega(B ; F)=\bigcap_{N>0} \operatorname{cl}\left(\bigcup_{n>N} F^{n}(B)\right)
$$

$$
\alpha(B)=\alpha(B ; F)=\bigcap_{N>0} \operatorname{cl}\left(\bigcup_{n>N} F^{-n}(B)\right) .
$$

If either $F$ or $F^{-1}$ is multi-valued, then we may wish to speak of the $\alpha$ - or $\omega$-limit set of a specific solution. Let $\sigma: \mathbf{Z}^{+} \rightarrow X$ be a full forward solution for $x \in X$. The $\omega$-limit set of $\sigma$ is defined to be

$$
\omega(\sigma)=\bigcap_{N>0} \operatorname{cl}\left(\bigcup_{n>N}\{\sigma(n)\}\right) .
$$

Similarly, if $\sigma: \mathbf{Z}^{-} \rightarrow X$ is a full backward solution for $x \in X$ the $\alpha$-limit set is

$$
\alpha(\sigma)=\bigcap_{N>0} \operatorname{cl}\left(\bigcup_{n>N}\{\sigma(-n)\}\right) .
$$

Consider an isolated invariant set $S$ with isolating neighborhood $N$. We say that a set $A \subset S$ is an attractor in $S$ if there exists a compact neighborhood $U \subset N$ of $A$ such that $\omega\left(U \cap S ; F_{S}\right)=A$. Any such neighborhood $U$ of $A$ is called an attracting neighborhood of $A$. Notice that in the definition we use the restriction map $F_{S}$. If we did not then it may be the case that $\omega(U \cap S)$ would not be contained in $S$.

The dual repeller to $A$ is defined to be

$$
A^{*}:=\{x \in S: \exists \text { solution } \sigma: \mathbf{Z} \rightarrow S \text { for } x \text { such that } \omega(\sigma) \cap A=\emptyset\} .
$$

If $F\left(A^{*}\right) \cap A=\emptyset$ then $\left(A, A^{*}\right)$ is called an attractor-repeller decomposition of $S$. The connecting orbits are

$$
C\left(A^{*}, A ; S\right):=\left\{x \in S: \exists \text { solution } \sigma_{x}: \mathbf{Z} \rightarrow S \text { with } \omega\left(\sigma_{x}\right) \subset A, \alpha\left(\sigma_{x}\right) \subset A^{*}\right\} .
$$

Again, notice that if $F$ is single-valued then the definitions coincide with the usual definitions. But, also notice that unlike the homeomorphism case, it may happen that $C\left(A^{*}, A ; S\right) \cap A \neq \emptyset$ or $C\left(A^{*}, A ; S\right) \cap A^{*} \neq \emptyset$. In particular, it may be the case that $F_{N}^{n}\left(A^{*}\right) \cap A \neq \emptyset$ for $n>1$. We do have the following useful equivalent definition for $(A, R)$ an attractor-repeller decomposition of $S$.

Proposition 7. $(A, R)$ is an attractor-repeller decomposition of $S$ if and only if $A, R \subset S$ are isolated invariant sets with $F(R) \cap A=\emptyset$ and with the following property. If $x \in S$ and $\sigma: \mathbf{Z} \rightarrow S$ is a full solution for $x$ then

1. $\sigma(\mathbf{Z}) \subset A$, or

2. $\sigma(\mathbf{Z}) \subset R$, or

3. $\omega(\sigma) \subset A$ and $\alpha(\sigma) \subset R$.

REMARK 8. If $F$ is a dmds and we take the stronger definition of an isolating neighborhood (see Remark 3) then the definitions of attractor and dual repeller are in fact dual. That is, if $(A, R)$ is an attractor-repeller decomposition of $S$ for $F$ then $(R, A)$ is an attractor-repeller decomposition of $S$ for $F^{-1}$. 
One may question whether there is a relationship between the Conley indices of $A$, $R$ and $S$. To answer this question we must find index pairs for each of the three isolated invariant sets. We make the following definition.

Definition 9. An index triple for $(A, R)$ is a compact triple $\left(N_{2}, N_{1}, N_{0}\right)$ with the property that $\left(N_{2}, N_{0}\right),\left(N_{2}, N_{1}\right)$ and $\left(N_{1}, N_{0}\right)$ are index pairs for $S, R$ and $A$ respectively.

The following theorem was proved for single-valued maps in [R1] and for multi-valued maps in [R2].

THEOREM 10. Let $S$ be an isolated invariant set and $(A, R)$ be an attractor-repeller decomposition of $S$. Then there exists an index triple for $(A, R)$.

Let $\left(N_{2}, N_{1}, N_{0}\right)$ be an index triple for $S$. Clearly the following diagram commutes, where $i, \iota$ and $\rho$ are defined by inclusions.

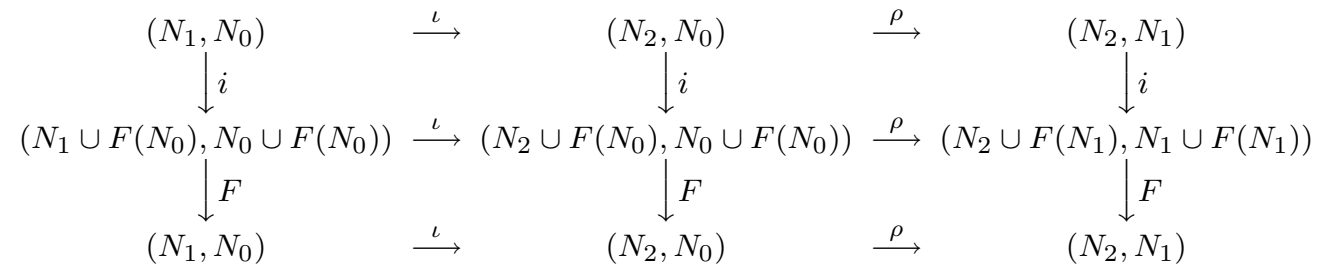

Passing to cohomology and applying the Leray functor one obtains the following result.

Proposition 11. If $(A, R)$ is an attractor-repeller decomposition of an isolated invariant set $S$ then we have the following long exact sequence of vector spaces and automorphisms

$$
\stackrel{\delta^{*}}{\longrightarrow} \mathrm{Con}^{k}(R) \longrightarrow \mathrm{Con}^{k}(S) \longrightarrow \mathrm{Con}^{k}(A) \stackrel{\delta^{*}}{\longrightarrow} \mathrm{Con}^{k+1}(R) \longrightarrow .
$$

The additive property of the Conley index gives the following useful corollary and its contrapositive.

Corollary 12. If $S=A \cup R$ then $\delta^{*}=0, C H^{*}(S) \cong C H^{*}(A) \oplus C H^{*}(R)$ and $\chi^{*}(S)$ is conjugate to $\chi^{*}(A) \oplus \chi^{*}(R)$.

Corollary 13. If $\delta^{*} \neq 0, C H^{*}(S) \neq C H^{*}(A) \oplus C H^{*}(R)$ or $\chi^{*}(S)$ is not conjugate to $\chi^{*}(A) \oplus \chi^{*}(R)$, then $S \neq A \cup R$. That is, $C(R, A ; S) \neq \emptyset$.

Notice that Corollary 12 is an improvement over the flow case. In particular, there are simple examples of attractor-repeller decompositions for which $\delta^{*}=0$ (and thus $\left.C H^{*}(S) \cong C H^{*}(A) \oplus C H^{*}(R)\right)$ but for which $\chi^{*}(S)$ and $\chi^{*}(A) \oplus \chi^{*}(R)$ are not conjugate linear maps. Thus we know the existence of a connecting orbit. Algebraically such a situation is possible because short exact sequences of vector spaces and endomorphisms do not always split. We give the following example.

EXAMPLE 14. Suppose $f$ is a single-valued map of the plane such that the following compact neighborhoods, $N_{1}$ and $N_{2}$ get mapped as shown below. 


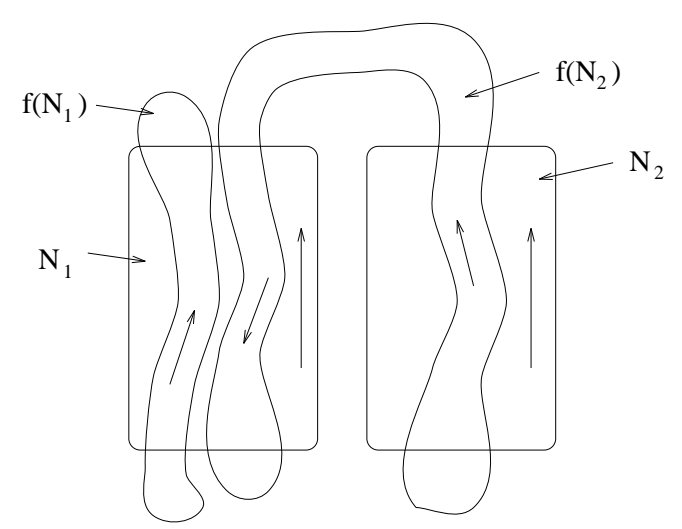

Let $A=\operatorname{Inv}\left(N_{1}\right), R=\operatorname{Inv}\left(N_{2}\right)$ and $S=\operatorname{Inv}\left(N_{1} \cup N_{2}\right)$. Clearly $(A, R)$ is an attractorrepeller decomposition of $S$. Furthermore, it is easy to see that

$$
\operatorname{Con}^{k}(A) \cong \operatorname{Con}^{k}(R) \cong\left\{\begin{array}{cc}
(\mathbf{Q}, i d) & k=1 \\
0 & \text { otherwise }
\end{array}\right.
$$

and

$$
\operatorname{Con}^{k}(S) \cong\left\{\begin{array}{cc}
\left(\mathbf{Q} \oplus \mathbf{Q},\left(\begin{array}{cc}
1 & 0 \\
-1 & 1
\end{array}\right)\right) & k=1 \\
0 & \text { otherwise }
\end{array} .\right.
$$

Notice that in this example $C H^{*}(S) \cong C H^{*}(A) \oplus C H^{*}(R)$ but since $\chi^{*}(S)$ and $\chi^{*}(A) \oplus$ $\chi^{*}(R)$ are not conjugate we conclude that there must exist a connecting orbit from $R$ to $A$. Furthermore, it is interesting to note that we are detecting a "degree 0 " connection. For instance, if we made the above example Morse-Smale, with $A$ and $R$ hyperbolic fixed point saddles, then we would be detecting the presence of a heteroclinic orbit.

Consider the graded vector space $D=C H^{*}(A) \oplus C H^{*}(R)$ and the linear map $\Delta=$ $\left(\begin{array}{cc}0 & 0 \\ \delta^{*} & 0\end{array}\right): D \rightarrow D$. We motivate the remainder of the paper with the following result.

Proposition 15 [R1]. $(D, \Delta)$ is a cochain complex with $H^{*}(D) \cong C H^{*}(S)$. Furthermore, there is a cochain map $d: D \rightarrow D$ such that the induced map on cohomology, $d^{*}$, is conjugate to $\chi^{*}(S)$.

Thus, we see that if one knows the Conley indices of both $A$ and $R$ and one knows the two matrices $\Delta$ and $d$ then one is able to reconstruct the Conley index of $S$.

4. Morse decompositions and index filtrations. We now consider the situation where an isolated invariant set can be decomposed into a finite number of attractors and repellers. Recall that a finite set $\mathcal{P}$ is called partially ordered by $<$ if

1. it is never the case that $p<p$ for $p \in \mathcal{P}$, and

2. if $p<q$ and $q<r$ then $p<r$.

3. If $p \neq q$ implies $p<q$ or $q<p$ then $\mathcal{P}$ is totally ordered. 
If $\mathcal{P}$ has a partial order $<$ then a subset $I \subset \mathcal{P}$ is called an interval provided $p<q<r$ and $p, r \in I$ implies $q \in I$. An interval $I$ is called attracting if $r<q$ and $q \in I$ implies $r \in I$. We will denote the set of intervals $\mathcal{I}=\mathcal{I}(\mathcal{P},<)$ and the set of attracting intervals $\mathcal{A}=\mathcal{A}(\mathcal{P},<)$. An ordered collection of intervals $\left(I_{1}, \ldots, I_{n}\right)$ is called an $n$-tuple provided $I_{1} \cup \ldots \cup I_{n} \in \mathcal{I}$ and $i<j, p \in I_{i}, q \in I_{j}$ implies $q \nless p$. We will denote this union by $I_{1} \ldots I_{n}$. The collection of $n$-tuples will be denoted $\mathcal{I}_{n}=\mathcal{I}_{n}(\mathcal{P},<)$.

Suppose $S$ is an isolated invariant set and $\mathcal{P}(<)$ is a finite, partially ordered set. Let $\mathcal{M}=\mathcal{M}(\mathcal{P},<)=\left\{M_{p} \subset S: p \in \mathcal{P}\right\}$ be any collection of mutually disjoint isolated invariant sets. For each interval $I \in \mathcal{I}$ define

$$
M_{I}:=\left(\bigcup_{p \in I} M_{p}\right) \cup\left(\bigcup_{p, q \in I} C\left(M_{p}, M_{q} ; S\right)\right) .
$$

Definition 16. A collection $\mathcal{M}=\mathcal{M}(\mathcal{P},<)=\left\{M_{p} \subset S: p \in \mathcal{P}\right\}$ of mutually disjoint isolated invariant sets is a Morse decomposition of $S$ if the following conditions hold:

1. $\left(M_{I}, M_{J}\right)$ is an attractor-repeller decomposition of $M_{I J}$ for all $(I, J) \in \mathcal{I}_{2}$,

2. for each $x \in S$ and each full solution for $x, \sigma: \mathbf{Z} \rightarrow S$, either $\sigma(\mathbf{Z}) \subset M_{p}$ for some $p$ or there exists $p, q \in \mathcal{P}$ such that $q<p$ and $\omega(\sigma) \subset M_{q}$ and $\alpha(\sigma) \subset M_{p}$, i.e. $x \in C\left(M_{p}, M_{q} ; S\right)$.

The sets $M_{p}$ are called Morse sets.

REMARK 17. For the single-valued case the definition of a Morse decomposition is slightly easier to state. In fact, one need only check that the second condition holds; for any $x \in S \backslash \bigcup_{p \in \mathcal{P}} M_{p}$ there exists $p, q \in \mathcal{P}$ such that $q<p$ and $x \in C\left(M_{p}, M_{q} ; S\right)$.

Given a collection of Morse sets it may be the case that there are several different orderings which may be placed on the decomposition. For sure, there is a minimal such ordering, the one defined by the connecting orbits. This ordering is called the $F$ defined ordering and will be denoted $<_{F}$. Any other ordering $<$ is a refinement of $<_{F}$. That is, $p<_{F} q$ implies $p<q$. We shall call any refinement of $<_{F}$ an admissible order.

We now have a large collection of isolated invariant sets. We would like to be able to compute Conley indices for each of them. In particular, we need index pairs. We state the following definition.

Definition 18. A collection of compact sets $\mathcal{N}=\mathcal{N}(\mathcal{P},<)=\{N(I): I \in \mathcal{A}(\mathcal{P})\}$ is called an index filtration for the Morse decomposition $\mathcal{M}(\mathcal{P},<)$ provided

1. for each $I \in \mathcal{A},(N(I), N(\emptyset))$ is an index pair for $M_{I}$,

2. for any $I, J \in \mathcal{A}$ we have $N(I) \cap N(J)=N(I \cap J)$ and $N(I) \cup N(J)=N(I \cup J)$.

TheOREM 19 [R2]. Suppose $F \in \mathcal{F}_{0}(X)$ and $S$ is an isolated invariant set. If $\mathcal{M}(\mathcal{P},<)$ $=\left\{M_{p}: p \in \mathcal{P}\right\}$ is a Morse decomposition of $S$ then there exists an index filtration $\mathcal{N}(\mathcal{P},<)=\{N(I): I \in \mathcal{A}(\mathcal{P})\}$ for $\mathcal{M}$.

We conclude this section with a remark which will prove useful in subsequent sections. Suppose $G \in \mathcal{F}(X)$ is a selector for $F \in \mathcal{F}(X)$. Also suppose $F$ has an iso- 
lated invariant set $S_{F}$ with Morse decomposition $\mathcal{M}_{F}(\mathcal{P},<)=\left\{M_{p, F}: p \in \mathcal{P}\right\}$. For each $p \in \mathcal{P}$ let $N_{p}$ be an isolating neighborhood for $M_{p, F}$. Define a new collection $\mathcal{M}_{G}(\mathcal{P},<)=\left\{M_{p, G}: p \in \mathcal{P}\right\}$ by $M_{p, G}=\operatorname{Inv}\left(N_{p}, G\right)$. Clearly, since $G$ is a selector for $F \mathcal{M}_{G}$ is a Morse decomposition of $S_{G}=\operatorname{Inv}(N, G)$. We call $\mathcal{M}_{G}$ the restriction of $\mathcal{M}_{F}$ by $G$. If $\mathcal{N}(\mathcal{P},<)$ is an index filtration for $F$ then it is also an index filtration for $G$.

5. Connection matrix pairs. In this section we present the definition of connection matrix pairs. They were introduced by the author for single-valued maps in [R1] and for multi-valued maps in [R2]. In fact, all the results given below and their proofs are identical for both the multi-valued and the single-valued cases. In this section we will assume that $S$ is an isolated invariant set, $\mathcal{P}(<)$ is a finite partially ordered set and $\mathcal{M}(\mathcal{P},<)$ is a Morse decomposition of $S$ with index filtration $\mathcal{N}(\mathcal{P},<)$. We denote the Conley index of $M_{I}$ by $\operatorname{Con}^{*}\left(M_{I}\right)=\left(C H^{*}(I), \chi^{*}(I)\right)$. As we mentioned earlier, we will assume that all coefficients lie in some field.

Below we define and state the existence of connection matrix pairs. In order to define them we must present a formidable collection of definitions. Perhaps the reader would benefit from an informal definition at this point. The connection matrix pair $(\Delta, d)$ consists of a pair of matrices of linear maps acting on the vector space $C \Delta(\mathcal{P})=\bigoplus_{p \in \mathcal{P}} C H^{*}(p)$. This pair will have the following properties. If $\Delta(I)$ and $d(I)$ are the restrictions of $\Delta$ and $d$ to $C \Delta(I)$ then

1. $\Delta(I)$ is a coboundary map and $d(I)$ is a cochain map,

2. there exists an isomorphism $\psi^{*}(I): H^{*}(C \Delta(I)) \rightarrow C H^{*}(I)$,

3. the induced map $d^{*}$ on $H^{*}(C \Delta(I))$ is conjugate to $\chi^{*}(I)$ via $\psi^{*}(I)$, i.e.

$$
\psi^{*}(I) d^{*}=\chi^{*}(I) \psi^{*}(I)
$$

Thus, knowing the Conley indices $\operatorname{Con}^{*}\left(M_{p}\right)$ and the connection matrix pair $(\Delta, d)$ we are able to reconstruct $\operatorname{Con}^{*}\left(M_{I}\right)$ for every interval $I$.

By Proposition 11 we know that attractor-repeller pairs fit together nicely in a long exact sequence. In the discussion that follows we shall see that Morse decompositions have a similarly nice relationship.

Definition 20. A cochain complex braid with endomorphism is a collection $\mathcal{C}=$ $\{(C(I), \partial(I), c(I)): I \in \mathcal{I}\}$ of cochain complexes and endomorphisms satisfying the following conditions.

1. For each $I \in \mathcal{I}$, there exists a cochain complex $C(I)$ and a cochain endomorphism $c(I): C(I) \rightarrow C(I)$ of degree 0 .

2. For all $(I, J) \in \mathcal{I}_{2}$ there exist chain maps $\iota(I, I J)$ and $\rho(I J, J)$ such that the following sequence is exact:

$$
0 \longrightarrow(C(J), c(J)) \stackrel{\rho}{\longrightarrow}(C(I J), c(I J)) \stackrel{\iota}{\longrightarrow}(C(I), c(I)) \longrightarrow 0 .
$$

3. For all $(I, J, K) \in \mathcal{I}_{3}$ the following braid diagram commutes. 


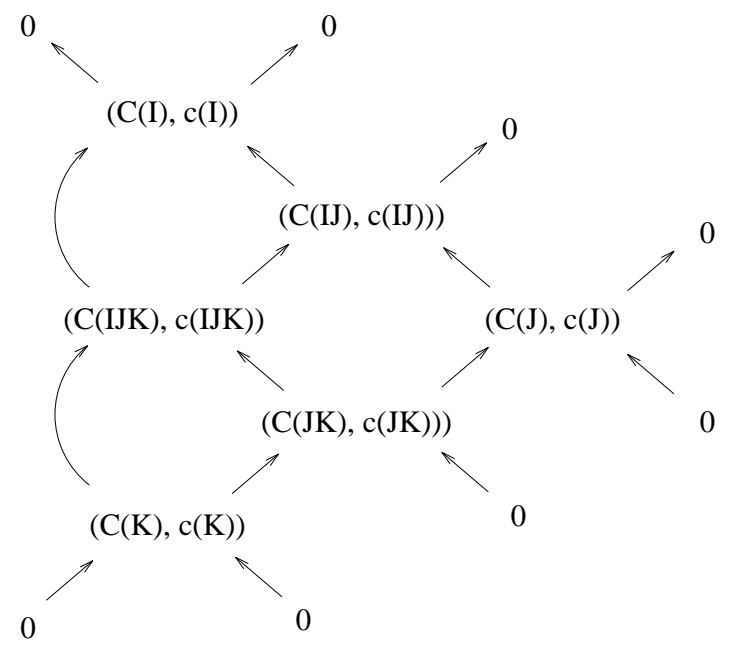

DeFINITION 21. A graded module braid with endomorphism is a collection $\mathcal{H}=$ $\{(H(I), a(I)): I \in \mathcal{I}\}$ of graded modules and linear endomorphisms such that the following conditions are satisfied:

1. For each $I \in \mathcal{I}$, there exists a graded module $H(I)$ and a linear endomorphism $a(I): H(I) \rightarrow H(I)$ of degree 0,

2. for all $(I, J) \in \mathcal{I}_{2}$, there exists a long exact sequence of modules and endomorphisms

$$
\stackrel{\delta^{n-1}}{\longrightarrow}\left(H^{n}(J), a^{n}(J)\right) \stackrel{\rho^{n}}{\longrightarrow}\left(H^{n}(I J), a^{n}(I J)\right) \stackrel{\iota^{n}}{\longrightarrow}\left(H^{n}(I), a^{n}(I)\right) \stackrel{\delta^{n}}{\longrightarrow},
$$

3. for all $(I, J, K) \in \mathcal{I}_{3}$ the following braid diagram commutes

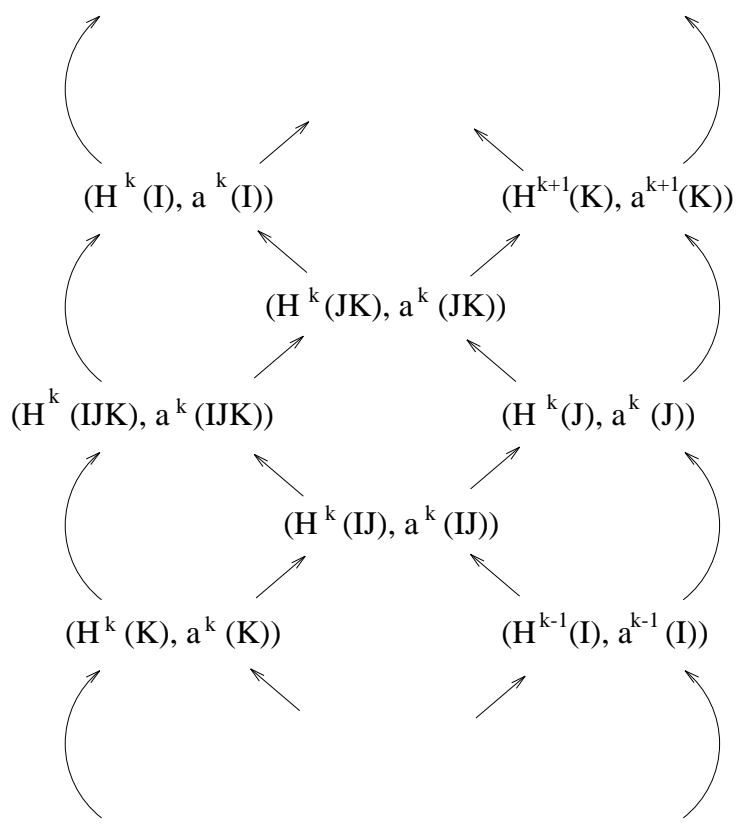


Proposition 22. Let $\mathcal{C}$ be a cochain complex braid with endomorphism. Passing to cohomology one obtains a graded module braid with endomorphism which we denote $\mathcal{H C}$.

Definition 23. Let $\mathcal{H}(\mathcal{P},<)=\{(H(I), a(I)): I \in \mathcal{I}\}$ and $\mathcal{H}^{\prime}(\mathcal{P},<)=\left\{\left(H^{\prime}(I)\right.\right.$, $\left.\left.a^{\prime}(I)\right): I \in \mathcal{I}\right\}$ be graded module braids with endomorphisms. Define a graded module braid homomorphism $\Psi: \mathcal{H} \rightarrow \mathcal{H}^{\prime}$ to be a collection of homomorphisms $\psi(I): H(I) \rightarrow$ $H^{\prime}(I)$ defined for all $I \in \mathcal{I}$ such that for $(I, J) \in \mathcal{I}_{2}$ the following diagram commutes.

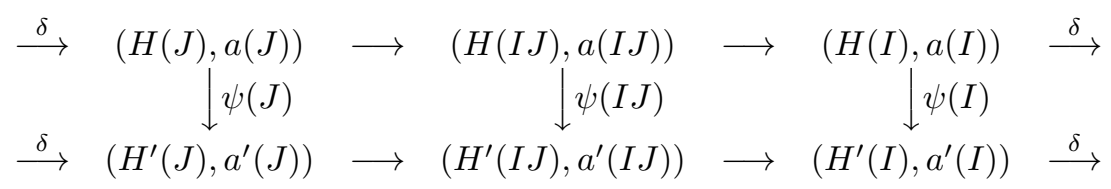

We say that $\Psi$ is an isomorphism if each $\psi(I)$ is an isomorphism.

We have at hand one example of a graded module braid. From the index filtration $\mathcal{N}(\mathcal{P},<)$ for the Morse decomposition $\mathcal{M}(\mathcal{P},<)$ we obtain the graded module braid

$$
\mathcal{H}(\mathcal{M}):=\left\{\left(C H^{*}(I), \chi^{*}(I)\right): I \in \mathcal{I}\right\} .
$$

The purpose of connection matrix pairs is to reconstruct this braid.

Let $C \Delta(I):=\bigoplus_{p \in I} C H^{*}(p)$ for $I \in \mathcal{I}$. We may regard any linear map $B: C \Delta(\mathcal{P}) \rightarrow$ $C \Delta(\mathcal{P})$ as a matrix of linear maps, $B=\left(B_{p q}\right)_{p, q \in \mathcal{P}}$ where $B_{p q}: C H^{*}\left(M_{q}\right) \rightarrow C H^{*}\left(M_{p}\right)$. Furthermore, we may define the submatrix $B(I): C \Delta(I) \rightarrow C \Delta(I)$ to be $B(I)=$ $\left(B_{p q}\right)_{p, q \in I}$. Such a map $B$ is called lower triangular if $q \not \leq p$ implies $B_{p q}=0$ and is called strictly lower triangular if $q \nless p$ implies $B_{p q}=0 . B$ is a coboundary operator if it is of degree +1 and $B^{2}=0$.

Suppose $\Delta: C \Delta(\mathcal{P}) \rightarrow C \Delta(\mathcal{P})$ is a strictly lower triangular coboundary operator and $d: C \Delta(\mathcal{P}) \rightarrow C \Delta(\mathcal{P})$ is a lower triangular cochain map (with respect to $\Delta$ ) of degree 0 . Let $\mathcal{C} \Delta(\mathcal{M})=\{(C \Delta(I), \Delta(I), d(I)): I \in \mathcal{I}\}$. Then it is clear that $\mathcal{C} \Delta(\mathcal{M})$ is a cochain complex braid with endomorphism. Passing to cohomology one obtains a graded module braid with endomorphism $\mathcal{H} \Delta(\mathcal{M})$.

We are finally in a position to make the following definition.

Definition 24. The pair of matrices $(\Delta, d)$ is called a connection matrix pair for the Morse decomposition $\mathcal{M}(\mathcal{P},<)$ if the graded module braid with endomorphism $\mathcal{H} \Delta(\mathcal{M})$ is isomorphic to the graded module braid with endomorphism $\mathcal{H}(\mathcal{M})$.

Let $\mathcal{C} \mathcal{M}(\mathcal{M}(\mathcal{P},<))$ denote the set of connection matrix pairs for the Morse decomposition $\mathcal{M}(\mathcal{P},<)$.

THEOREM 25 [R2]. For any isolated invariant set $S$ and any partially ordered Morse decomposition $\mathcal{M}(\mathcal{P},<)$ of $S, \mathcal{C} \mathcal{M}(\mathcal{M}(\mathcal{P},<)) \neq \emptyset$.

Just as is the case for connection matrices for flows, there may not exist a unique connection matrix pair for a given Morse decomposition. Understanding this nonuniqueness is not yet complete. It seems that nonuniqueness is a consequence of the continuation property.

6. Continuation of connection matrix pairs. One of the crucial properties of the Conley index is that it is robust under small perturbations of the map; it has the 
so-called continuation property. In [R1] we developed the theory of connection matrix pairs for continuous maps of locally compact metric spaces. In [R2] we show that, like the rest of the Conley index theory, connection matrix pairs satisfy the continuation property.

Let $F:[a, b] \times \mathscr{P}(X) \rightarrow \mathscr{P}(X)$ be a continuous homotopy through $\mathcal{F}(X)$. That is, for each $\lambda \in[a, b], F_{\lambda} \in \mathcal{F}(X)$ where $F_{\lambda}(A)=F(\lambda, A)$. Assume that $N$ is an isolating neighborhood for $F_{\lambda}$ for all $\lambda \in[a, b]$. Let $S_{\lambda}=\operatorname{Inv}\left(N, F_{\lambda}\right)$. Furthermore, suppose $S_{a}$ and $S_{b}$ have Morse decompositions $\mathcal{M}\left(\mathcal{P},<, F_{a}\right)$ and $\mathcal{M}\left(\mathcal{P},<, F_{b}\right)$ respectively. We say that $\mathcal{M}\left(\mathcal{P},<, F_{a}\right)$ and $\mathcal{M}_{b}\left(\mathcal{P},<, F_{b}\right)$ are related by continuation if each $S_{\lambda}$ has a Morse decomposition $\mathcal{M}\left(\mathcal{P},<, F_{\lambda}\right)$ and if there exists a collection of sets $\mathcal{N}$ which serves as an index filtration for each $\mathcal{M}_{\lambda}$. By Proposition $6 \mathcal{N}$ is also an index filtration for any selector $G_{a}$ of $F_{a}$ and any selector $G_{b}$ of $F_{b}$, thus we will say that $\mathcal{M}\left(\mathcal{P},<, G_{a}\right)$ and $\mathcal{M}\left(\mathcal{P},<, G_{b}\right)$ are related by continuation. We then make this definition transitive, that is, if $\mathcal{M}_{b}$ is related by continuation to both $\mathcal{M}_{a}$ and $\mathcal{M}_{c}$ we say that $\mathcal{M}_{a}$ and $\mathcal{M}_{c}$ are related by continuation. Thus we obtain an equivalence relation.

If $<$ is the $F_{a}$-defined order on a Morse decomposition $\mathcal{M}_{a}$, and $\mathcal{M}_{b}$ is a Morse decomposition related to $\mathcal{M}_{a}$ by continuation, then $<$ may not be the $F_{b}$-defined order. Of course, it does follow that $<$ is an admissible order for $\mathcal{M}_{b}$.

The proof of the continuation property of connection matrix pairs can be broken into two parts. First we show that if two Morse decompositions are related by continuation then they have the same set of connection pairs. This statement is easy to prove and is stated below in Proposition 26. Second, we show that in fact Morse decompositions always continue to Morse decompositions for nearby maps. We shall sketch the proof of Proposition 29 below.

Proposition 26. Suppose $F_{a}, F_{b} \in \mathcal{G}(X)$ and the Morse decompositions $\mathcal{M}_{a}(\mathcal{P},<)$ and $\mathcal{M}_{b}(\mathcal{P},<)$ are related by continuation. Then $\mathcal{C} \mathcal{M}\left(\mathcal{M}_{a}(\mathcal{P},<)\right)=\mathcal{C} \mathcal{M}\left(\mathcal{M}_{b}(\mathcal{P},<)\right)$. That is, $(\Delta, d)$ is a connection matrix pair for $\mathcal{M}_{a}$ if and only if it is a connection matrix pair for $\mathcal{M}_{b}$.

REMARK 27. Recall that the collection of connection matrix pairs depends on the choice of ordering on the Morse sets. If one were to refine an ordering $\mathcal{P}(<)$ to obtain $\mathcal{P}\left(<^{\prime}\right)$ then $\mathcal{P}\left(<^{\prime}\right)$ would have fewer intervals. Thus there would be fewer algebraic restrictions on the set of connection matrix pairs, and we have $\mathcal{M}(\mathcal{P},<) \subset \mathcal{M}\left(\mathcal{P},<^{\prime}\right)$. In particular, if $<_{a}$ is the map defined order for $F_{a}$ and $\mathcal{M}_{a}(\mathcal{P},<)$ and $\mathcal{M}_{b}(\mathcal{P},<)$ are related by continuation, then $\mathcal{C M}\left(\mathcal{M}_{b}\left(\mathcal{P},<_{a}\right)\right)=\mathcal{C M}\left(\mathcal{M}_{a}\left(\mathcal{P},<_{a}\right)\right)$, but $\mathcal{C M}\left(\mathcal{M}_{b}\left(\mathcal{P},<_{b}\right.\right.$ )$) \subset \mathcal{C M}\left(\mathcal{M}_{a}\left(\mathcal{P},<_{a}\right)\right)$.

Suppose $F \in \mathcal{F}_{0}(X)$ and that $\mathcal{M}_{0}(\mathcal{P},<)$ is a Morse decomposition of an isolated invariant set $S_{0}=\operatorname{Inv} N$. Let $G_{\lambda}$ be the fattening homotopy of $F$. For $\lambda$ small enough, say $\lambda \in[0,2 \varepsilon), N$ is an isolating neighborhood for $G_{\lambda}$.

Lemma 28. Let $G_{\lambda}$ be the fattening homotopy for $F \in \mathcal{F}_{0}(X)$. For $\varepsilon>0$ small enough, $\mathcal{M}_{0}(\mathcal{P},<)$ continues to a Morse decomposition $\mathcal{M}_{\varepsilon}(\mathcal{P},<)$ for $G_{\varepsilon}$.

This statement seems weaker than the one we need, but we see that it will suffice. Let $\varepsilon>0$ be the one of Lemma 28. Then there exists an index filtration $\mathcal{N}(\mathcal{P},<)$ for the 
$\varepsilon$-fattening map $G_{\varepsilon}$. Since $\mathcal{N}$ is also an index filtration for any selector of $G_{\varepsilon}$, Proposition 6 gives us the following result.

Proposition 29. For $G \in \mathcal{F}_{0}(X)$ sufficiently close to $F$, say $G(x) \subset B_{\varepsilon}(F(x))$ for all $x \in X$, the Morse decomposition $\mathcal{M}_{F}(\mathcal{P},<)$ continues to a Morse decomposition $\mathcal{M}_{G}(\mathcal{P},<)$.

We combine Proposition 26 and Proposition 29 to obtain the following lemma.

Lemma 30 (Stable index filtrations). Let $F \in \mathcal{F}_{0}(X)$ and let $S$ be an isolated invariant set with Morse decomposition $\mathcal{M}_{F}(\mathcal{P},<)$. Then there exists an index filtration $\mathcal{N}(\mathcal{P},<)$ which is stable under small perturbations in $\mathcal{F}(X)$. That is, there exists $\varepsilon>0$ such that if $G \in \mathcal{F}(X)$ has the property $G(x) \subset B_{\varepsilon}(F(x))$ for all $x \in X$ then $\mathcal{N}$ is an index filtration for $G$.

Theorem 31 (Continuation of connection matrix pairs). Let $F \in \mathcal{G}_{0}(X)$ and let $S$ be an isolated invariant set with Morse decomposition $\mathcal{M}_{F}(\mathcal{P},<)$. Then for $G \in \mathcal{G}(X)$ sufficiently close to $F$ the graded module braids with endomorphisms $\mathcal{H}\left(\mathcal{M}_{F}\right)$ and $\mathcal{H}\left(\mathcal{M}_{G}\right)$ are isomorphic. In particular $\mathcal{C M}\left(M_{a},<\right)=\mathcal{C} \mathcal{M}\left(\mathcal{M}_{b},<\right)$.

7. Computer assisted proofs. We conclude with an application to numerical analysis. One would like to be able to model continuous maps $f: \mathbf{R}^{m} \rightarrow \mathbf{R}^{m}$ on a computer in such a way that Morse decompositions and connection matrix pairs could be computed. Of course any such model must take the form of a multi-valued map. In particular, such a map may be upper semicontinuous. Theorem 30 was only proved for continuous maps, thus we must proceed with care.

Suppose $f: \mathbf{R}^{m} \rightarrow \mathbf{R}^{m}$ is an $L$-Lipschitz continuous map and $N \subset \mathbf{R}^{m}$ is a compact neighborhood. Suppose $N_{n} \subset N$ is a family of subsets with the property that for any $x \in N$ we have $\operatorname{dist}\left(x, N_{n}\right)<\frac{1}{n}$. Moreover, suppose that for any $x \in N_{n}$ we can find numerically an approximation $f_{n}(x)$ such that $\operatorname{dist}\left(f(x), f_{n}(x)\right) \leq \frac{1}{n}$. We now define a multi-valued map by

$$
F_{n}(x)=\operatorname{conv} \bigcup\left\{B_{r}\left(f_{n}(y)\right): \operatorname{dist}(x, y)=\operatorname{dist}\left(x, N_{n}\right)\right\}
$$

where $r=(1+L) / n$.

Suppose that $N$ is an isolating neighborhood for $f$ and $\mathcal{M}(\mathcal{P},<)$ is a Morse decomposition for $S=\operatorname{Inv} N$. Let $N_{I}$ be an isolating neighborhood for the set $M_{I}$. For each $n$ define $M_{I, n}=\operatorname{Inv}\left(N_{I}, F_{n}\right)$. Let $\mathcal{M}_{n}(\mathcal{P},<)=\left\{M_{p, n}: p \in \mathcal{P}\right\}$.

Let $\varepsilon>0$ be small enough so that the Morse decomposition $\mathcal{M}$ continues to the $\varepsilon$-fattening map $G_{\varepsilon}$. Now, if we take $n$ large enough $F_{n}$ is a selector for $G_{\varepsilon}$, thus $\mathcal{M}_{n}$ is a Morse decomposition for $F_{n}$. Lastly, we see that the index filtration for $G_{\varepsilon}$ is an index filtration for both $f$ and $F_{n}$. This argument proves the following theorem.

TheOrem 32 [R2]. For $n$ large enough $\mathcal{M}_{n}(\mathcal{P},<)$ is a Morse decomposition of $\operatorname{Inv}\left(N, F_{n}\right)$. Moreover, there exists an index filtration $\mathcal{N}$ for both $f$ and $F_{n}$. Thus, $\mathcal{C} \mathcal{M}(\mathcal{M})$ $=\mathcal{C M}\left(\mathcal{M}_{n}\right)$. 


\section{References}

[C] C. Conley, Isolated Invariant Sets and the Morse Index, CBMS Reg. Conf. Ser. in Math. 38, AMS, Providence, 1978.

[Fr] J. Franks, Homology and Dynamical Systems, CBMS Reg. Conf. Ser. in Math. 49, AMS, Providence, 1982.

[F1] R. Franzosa, The Connection Matrix Theory for Morse Decompositions, Trans. AMS 311 (1989), 561-592.

[F2] R. Franzosa, The Continuation Theory For Morse Decompositions and Connection Matrices, Trans. AMS 310 (1988), 781-803.

[FM] R. Franzosa and K. Mischaikow, The Connection Matrix Theory for Semiflows on (Not Necessarily Locally Compact) Metric Spaces, J. Differential Equations 71 (1988), 270-287.

[G1] L. Górniewicz, Homological Methods in Fixed Point Theory of Multi-valued Maps, Dissertationes Mathematicae 129 (1976).

[G2] L. Górniewicz, Topological Degree of Morphisms and its Applications to Differential Inclusions, Raccolta di Seminari del Dipartimento di Matematica dell'Università degli Studi della Calabria 5 (1983).

[KM1] T. Kaczynski and M. Mrozek, Conley Index for Discrete Multi-valued Dynamical Systems, Top. App. 65 (1995), 83-96.

[KM2] T. Kaczynski and M. MrozeK, Stable Index Pairs for Discrete Dynamical Systems, preprint, 1994.

[R1] D. Richeson, Connection Matrix Pairs for the Discrete Conley Index, preprint.

[R2] D. Richeson, Morse Decompositions and Connection Matrix Pairs For Multi-valued Maps, preprint. 